\title{
Estimation of thermal diffusivity mapping of CMC composites during repeated tensile tests: Several considerations about a suitable processing of the temperature field related to rear face flash experiments
}

\author{
by J. El Yagoubi*, J. Lamon** and J.C. Batsale ${ }^{\star \star *}$
}

\begin{abstract}
*LCTS, University of Bordeaux, 3 Allée de la Boétie, 33600 Pessac, France, elyagoubi@/cts.u-bordeaux1.fr **MATEIS, INSA Lyon, 7 Avenue Jean Capelle, 69621 Villeurbanne, France, jacques.lamon@insa-lyon.fr **TREFLE, ENSAM Bordeaux, Esplanade des Arts et Métiers, 33405 Talence, France, jeanchristophe.batsale@ensam.eu
\end{abstract}

\begin{abstract}
Ceramic matrix composites (CMC) are very attractive materials for structural applications at high temperatures. Not only must CMC be damage tolerant, but they must also allow thermal management. For this purpose heat transfer must be controlled even in the presence of damage.
\end{abstract}

\section{Introduction}

Damage consists in multiple cracks that form in the matrix and ultimately in the fibers, when the stresses exceed the proportional limit. Therefore the thermal conductivity (or thermal diffusivity) dependence on applied load is a factor of primary importance for the design of CMC components [1].

In order to investigate such thermomechanical mechanisms, it is here proposed to adapt the suitable rear face flash methods in order to estimate the thermal diffusivity mapping of $\mathrm{CMC}$ samples during tensile tests.

\section{Transverse and In-plane thermal diffusivity}

This work proposes first to consider simple estimation methods for the transverse diffusivity mapping. Such methods consist in validating the implementation of 1D heat transfer models, in the case of uniform flash irradiation. Two processing methods (Singular Values Decomposition and Linear Least Squares from asymptotic expansion) are compared and validated in order to obtain a suitable tool for the processing of the large amount of data necessary for the characterisation of a CMC sample in systematic tensile tests. [2][3]

In order to estimate a global in-plane diffusivity, a second flash method with non-uniform irradiation is implemented. [4]

\section{Damage investigation through thermal measurement}

The material under consideration is a $2 \mathrm{D}$ woven multilayered $\mathrm{SiC} / \mathrm{SiC}$ composite that exhibit a typical non-linear mechanical behaviour attributed to multiple cracks and fibre ruptures. Monitoring the evolution of the thermal diffusivity is a very convenient way to investigate the damage in composite materials. The results given in the figure 1 and 2 depict the main characteristics of the behaviour of such samples during tensile tests.

From a global point of view, one can notice that the transverse and in plane thermal diffusivities are both decreasing versus the applied load (figure 2). The evolution of the transverse thermal diffusivity maps (figure 1) indicates that the damaged samples are less heterogeneous that the initially non damaged sample.

The comparison of the global in plane and traverse diffusivities (figure 2) is also an interesting example of information given by such methods. Here, the results are revealing the anisotropy of the damage. 


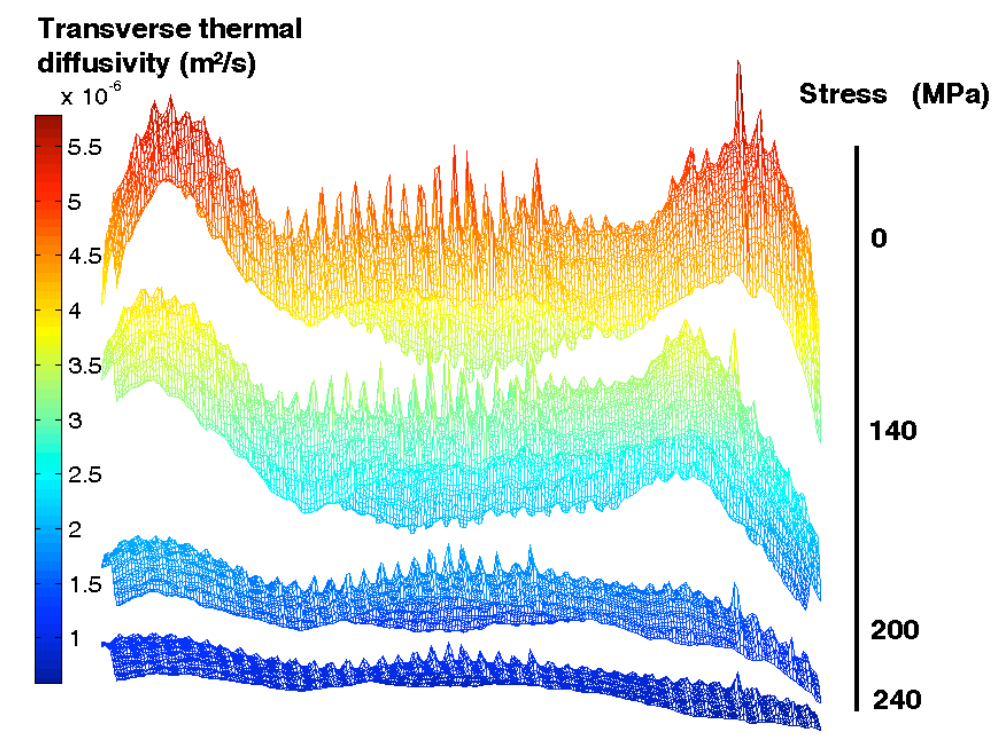

Fig. 1. Evolution of the transverse thermal diffusivity mapping versus the loading levels

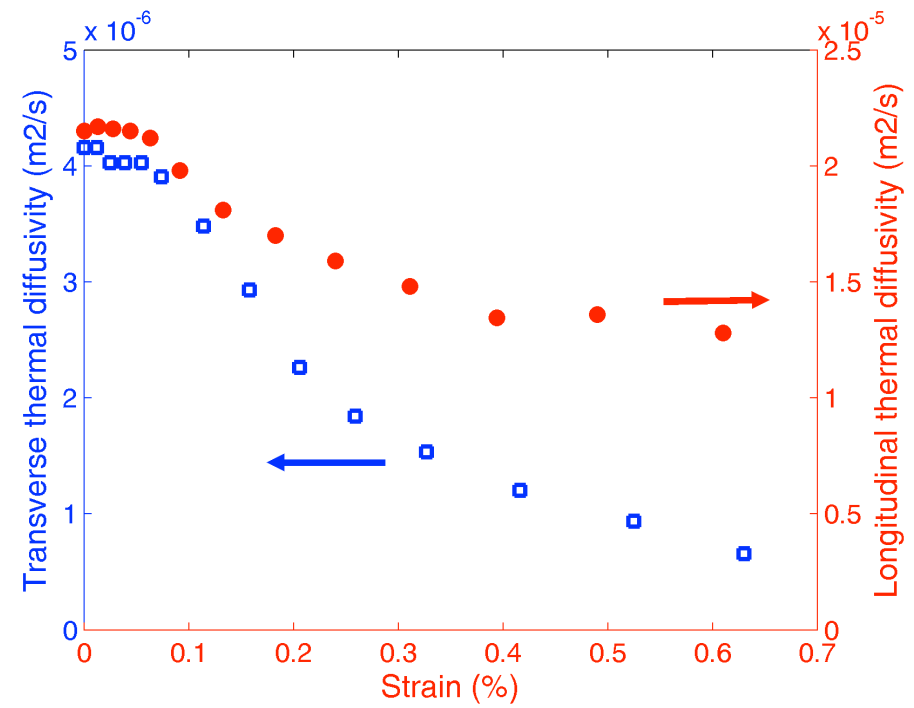

Fig. 2. Evolution of the global transverse and in-plane thermal diffusivity versus the strain

\section{REFERENCES}

[1] El Yagoubi J., "Effect of mechanical damage on the thermal properties of CMCs: multiscale approach". Doctorate thesis, University of Bordeaux, 2011.

[2] Mourand D., Gounot J., and Batsale J.C. "New sequential method to process noisy temperature response from flash experiment measured by infrared camera". Review of Scientific Instruments, 69: 1437-1440, 1998.

[3] Bamford M. "Méthode flash et thermographie infrarouge pour la cartographie de propriétés thermophysiques: application à la caractérisation en thermomécanique". Doctorate thesis, University of Bordeaux, 2007.

[4] Philippi I., Batsale J.C., Maillet D., and Degiovanni A.. "Measurement of thermal diffusivity through processing of infrared images". Review of Scientific Instruments, 66 :182-192, 1994. 